\title{
Role of tissue engineering in oral \& maxillofacial surgery - a review
}

\author{
Angeline L R. ${ }^{{ }^{*}}{ }$, Rohini T. ${ }^{2}$, Balasubramaniam . $^{3}$ \\ DOI: https://doi.org/10.17511/ijmrr.2019.i02.11 \\ 1* Reenu Angeline L, BDS, Department of Oral \& Maxillofacial Surgery, Government Dental College \& Hospital, Chennai, Tamil Nadu, India. \\ 2 Rohini T., Assistant Professor, Department of Oral \& Maxillofacial Surgery, Government Dental College \& Hospital, Chennai, Tamil Nadu, \\ India. \\ 3 Saravanan Balasubramaniam, Retd. Principal, Department of Oral \& Maxillofacial Surgery, Government Dental College \& Hospital, \\ Chennai, Tamil Nadu, India.
}

Tissue engineering can be defined as the "reconstitution of tissue and organs, in vitro for use as model systems in basic and applied research, or for use as grafts to replace damaged or diseased body parts or body functions". Biomaterials have been used as replacement tissues and grafts have been used to reconstruct defects in craniofacial region till Uristmade the first attempt of producing exogenous bone with the help of bone morphogenetic proteins. The success of tissue engineering over the field of all transplantation is that conceptually a three-dimensional functional tissue is designed. This field has become a boon to the Cranio Maxillofacial surgeons and has provided them with a supplement to existing treatment for reconstruction of Oral \& Craniofacial region. The purpose of this article is to present an overview of the various uses of tissue engineering in the field of Oral and Maxillofacial Surgery.

Keywords: Tissue Engineering, Scaffold, Growth factors, Signalling molecules

Corresponding Author

Reenu Angeline L, BDS, Department of Oral \& Maxillofacial Surgery, Government Dental College \& Hospital, Chennai, Tamil Nadu, India. Email: reenuangel $7777 @ g m a i l . c o m$

\section{How to Cite this Article}

Angeline LR, Rohini T, Balasubramaniam S. Role of tissue engineering in oral \& maxillofacial surgery - a review. Int J Med Res Rev. 2019;7(2):115-121. Available From

https://ijmrr.medresearch.in/index.php/ijmrr/article/ view/1050
To Browse

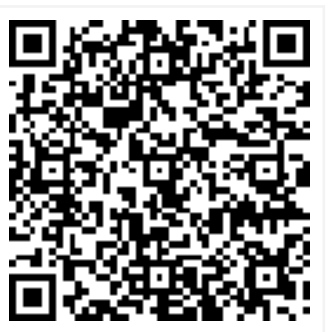

Manuscript Received 2019-04-03

Conflict of Interest No

Review Round 1
2019-04-12
Funding
Nil
(c) 2019 by Reenu Angeline L, Rohini T., Saravanan Balasubramaniam and Published by Siddharth Health Research and Social
Welfare Society. This is an Open Access article licensed under a Creative Commons Attribution 4.0 International License https://creativecommons.org/licenses/by/4.0/ unported [CC BY 4.0].

Accepted 2019-04-22

Note 


\section{Introduction}

Tissue engineering can be defined as the "reconstitution of tissue and organs, in vitro for use as model systems in basic and applied research, or for use as grafts to replace damaged or diseased body parts or body functions" [1]. The terms such as Regenerative medicine, reparative medicine, and tissue engineering have been used interchangeably to describe this novel breakthrough in the field of medicine. Biomaterials have been used as replacement tissues and grafts have been used to reconstruct defects in craniofacial region till Urist [2] made the first attempt of producing exogenous bone with the help of bone morphogenetic proteins.

Regenerative medicine is a multi and inter disciplinary field applying the principles of bio engineering for the development of biologic substitution that restore, maintain or improve tissue function [3]. Tissue engineering requires three basic tools: cells, a scaffold and signalling molecules to regenerate new tissues within a specific environment [3]. The success of tissue engineering over the field of all transplantation is that conceptually a three-dimensional functional tissue is designed. This field has become a boon to the Cranio Maxillofacial surgeons and has provided them with a supplement to existing treatment for reconstruction of Oral \& Craniofacial region such as the teeth, periodontium, bones, soft tissues (Oral mucosa, Conjunctiva, Skin), salivary gland and TMJ (Bone and Cartilage) blood vessels, muscle tendons and nerves.

The reconstruction of maxillofacial region has undergone a transition from non-vascularised bone grafts to micro vascular free flap. The fibula, anterior lateral thigh (ALT) and radial forearm free flaps are the most commonly used donor sites because of their ease in harvest, modest donor site morbidity, inclusion of large volume and variety of tissue types from a single vascular pedicle [4]. However, they have their own drawbacks such as original form of the bony defect cannot be established, difficulties in dental rehabilitation and there is a need for subsequent revision surgeries. These limitations have made the surgeons to move towards tissue engineering to enhance bone formation in large defects.

The purpose of this article is to present an overview of the various uses of tissue engineering in the field of Oral and Maxillofacial Surgery.

\section{Principles of Regenerative Medicine}

The three basic tools required for regeneration of tissues are cells, scaffold and signalling molecules. Regeneration of the tissue follow the pathway of repair such as inflammation, proliferation and remodelling. This process is completed through the biological signals which initiate the multiplication of cells and morphogenic signals which induce tissue specific differentiation (Fig. 1)

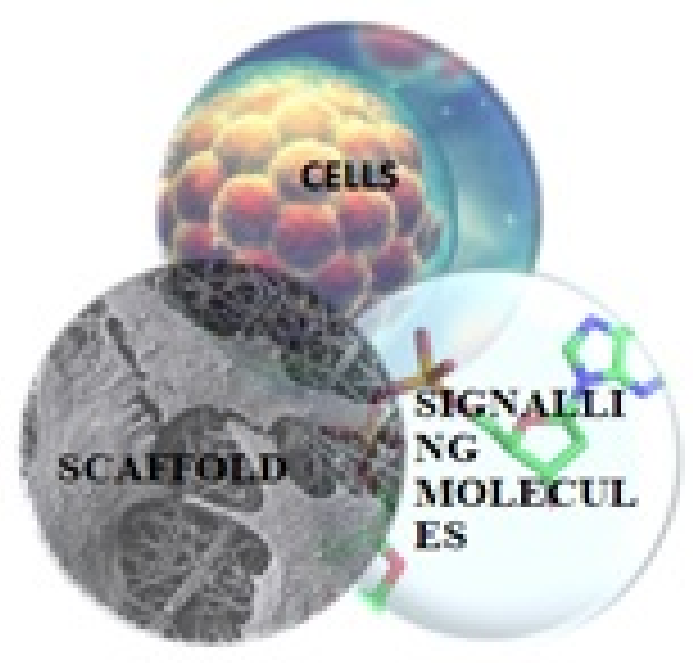

\section{Fig.-1: Triads of Tissue Engineering}

1.1: Signalling molecules- Growth factors and cytokines are conjugated to the ECM such as BMP (Bone Morphogenic Protein), fibroblast growth factor-2, Interleukin - 6, IGF (Insulin Growth Factor), PDGF (Platelet Derived Growth Factor), and TGF (Transforming Growth Factor) - $\beta 1$ [5]. This co localization acts as a storage pool of growth factors and reduces degradation of growth factors.

The growth factors originate from extracellular matrix (ECM) where they are stored and released during the process of remodelling and repair. Hence ECM serves as a scaffold for migration of cells and their arrangement in a tissue specific manner. By this cell, signalling molecules and ECM are interlinked for tissue regeneration in vivo.

1.2: Scaffold- When the cells and signalling molecules are transformed to the in vitro environment, the ECM is replaced by synthetic or natural scaffold to accommodate and arrange the cells in three-dimensional manner.

The scaffold will act as a template for cell proliferation, extracellular matrix deposition, tissue regeneration and remodelling. 
The ideal properties of a scaffold are, it should be biocompatible, should have adequate mechanical strength, undergo degradation, support cell interaction, since proliferation is anchorage dependent should provide surface for cell attachment, proliferation, differentiation and migration [6]. It must have adequate tensile and compressive strength pertaining to a particular functional environment. In vitro, it should have sufficient mechanical strength to withstand hydrostatic pressure and maintain the spaces required for cell ingrowth and matrix production. In vivo, mechanical properties of scaffold should match with the tissue to the regenerated.

The degradation rate should match with the regeneration rate of newly forming tissue and undergo nontoxic degradation. Porosity and interconnectivity are also important for effective diffusion of gases and nutrientsand should also allow efficient removal of waste products.

The optimal pore size suggested is between $200 \mu \mathrm{m}$ and $400 \mu \mathrm{m}$ [7]. Efforts are being made to increase the strength of solid phase while maintaining porosity in the range of $70 \%$ [8].

Scaffolds can be bone grafts, matrices, polymeric materials and composite scaffolds.

Bone grafts can be autologous, homologous, xenografts and alloplastic grafts ( $\beta$ TCP[tri-calcium phosphate], bio ceramics, HA [Hydroxyapatite]).

Matrices can be organic matrices which include collagen type I, inorganic matrices (HA and Calcium phosphate base matrices. CaP based inorganic matrices can further classified as ceramic [bio glass ceramics and HA ceramics] and cement matrices $[\beta$ $\mathrm{TCP}])$.

Polymeric materials can be classified as natural or synthetic. Organic polymers used as scaffold are alginate and hyaluronic acid, chitosan, peptide hydro gel and collagen. Synthetic polymers: PLA (Polylactic acid), PGA (Polyglycolic acid), PLGA (poly lactic-co-glycolic acid), PEG (Polyethylene glycol), PEG with PLGA, PCL (Polycaprolactone).

Composite scaffolds are scaffolds obtained by union of several components.

1.3: Cells- Cells are required for integration of materials used in reconstruction. Stem cell supplementation may work synergistically with natural progenitor cell in migration and differentiation.
Stem cells by definition are those cells with two fundamental characteristics such as self-renewal and potency. Stem cells are of 3 types (Adult Stem cells [ASCs], Embryonic Stem cells [ESCs] and induced pluripotent stem cells [iPSCs] [9].

Adult stem cells reside in fully differentiated tissue such as bone marrow and periosteum, teeth, dental pulp, supporting structures and gingival as well as fat, muscle, nervous tissues, skin and others. ESCs are usually isolated from the inner wall of the perimplantation blastocysts. These cells are more proliferative and pluripotent. iPSCs, a new source of pluripotent stem cells can be derived from adult cells by introducing 4 pleuripotency genes (Oct3/4, Sox2, cMyc and KIF4) which are also called YAMANAKA factors [10]. Craniofacial BMSCs (Bone Mesenchymal Stem Cells) proliferate faster, have increased levels of alkaline phosphatases and form higher levels of compact bone compared with long bone BMSCs. Dental pulp stem cells have the potential to regenerate dentin/pulp like complex. Periodontal ligament stem cells are capable of differentiating into cementoblast like cells, adipocytes and cells with capacity to form collagen. Stem cells from apical papilla, human exfoliated deciduous tooth have also been used for bio root engineering. Stem cells derived from gingival tissues have been reported to not only possess stem cell properties but also immunomodulatory and antiinflammatory effects in tissue. GMSCs (Gingival Mesenchymal Stem Cells) can be differentiated into adipocytes, osteocytes, chondrocytes and different lineages of neural cells such as neuronal and schwaan cells. Macrophages exhibit M2- like macrophage phenotypes characterized by an increase expression of mannose receptors and antiinflammatory cytokine IL 10 (Interleukin) while showing decreased expression of pro inflammatory cytokine, TNF-a. They are also capable of suppressing the activation and function of inflammatory Th-1 and Th-17 cells and promoting generation of anti-inflammatory regulatory $T$ cells.

\section{Bone Regeneration}

Three tissue engineering approaches exist to create bone [11]

01. Implantation of a scaffold that is conducive to bone tissue infiltration

02. Supplementation of a scaffold with bioactive molecules.

03. Implantation of cells in combination with scaffold. 
Another dimension of bone tissue engineering is the use of pre-fabricated bio engineered bone flaps. Warnke et al [12] reported a novel method of repairing a human mandible by in vivo tissue engineering. rhBMP-7 and bone marrow derived mesenchymal stem cells were added to a bioresorbable polylactic scaffold, which was loaded in a titanium mesh tray as an external scaffold.

The patient served as his own bioreactor, as titanium mesh was grown inside the patient's latissimus dorsi muscle. After seven weeks, the formed bone with titanium mesh was transplanted to the patient's mandible. The results showed greater osteoblastic activity and marked bone formation in all parts of the mandible during the 38th week.

Another important human trial aiming to maintain sustainable blood supply to the graft was conducted by using prefabricated tissue engineered graft which was transferred in pedicled flaps. Heliotis et al [13] demonstrated the preparation of vascularized bone flap extra skeletally with combination of osteogenetic protein (OP-1) and HA flap for mandibular reconstruction. 3 blocks of $\mathrm{HA}$ were adjusted to the shape of the mandibular body and ramus, OP-1was smeared over the blocks and then implanted within the pectoralis major muscle for 15 weeks.

The implanted composite was raised with attached muscle leaving it pedicled to thoracoacronial artery for mandibular reconstruction. The rationale behind using mesenchymal stem cells into scaffolds with /without PRP or BMP is to achieve osteogenesis, osteoconductivity, osteopromotion, and osteoinductivity. It has been reported that bone formed using this technique respond more to secondary surgical intervention such as distraction osteogenesis. Lee et al [14] reported successful reconstruction of $15 \mathrm{~cm}$ segmented mandibular defect using bone marrow stem cells.

\section{Soft Tissue Engineering}

The advantages of reconstructing craniomaxilla facial soft tissue defects using tissue engineering are minimal to no donor site morbidity, faster restoration and improvement of outcomes, enhancement in the quality, shape and function of the soft tissue being regenerated, autogenous nature of the fabricated soft tissue construct bypassing the need for immunosuppression. Reconstruction of oral mucosa consists of invitro fabrication followed by implantation in vivo.
Ex vivo produced oral mucosa equivalent (EVPOME) was first designed by Fienburg, Marculo, Izumi group at the university of Michigan [15].

The steps involved are

01. CULTURES: harvesting of cells from $5 \mathrm{~mm} 2$ punch biopsy from the patient, either keratinocytes or non-keratinocytes which are isolated, grown and purified in a defined medium on a decellularized cadaveric human dermis.

02. The mucocutaneous junction is created by growing both oral and epidermal keratinocytes on the same piece of dermal equivalent in separate compartments divided by a physical barrier made of inert biocompatible material such as PDMS (polydimethylsiloxane). The oral and skin keratinocytes are harvested from unattached oral mucosa and retro auricular area by punch biopsy. The intermediate area between skin and keratinocytes are devoid of cells.

03. GROWTH: The keratinocytes are allowed to proliferate and then differentiate by altering the concentration of calcium in the medium.

04. On removal of the (PDMS) barrier the oral and skin keratinocytes are allowed to co mingle in the unseeded acellular area and form a third site vermilion.

The 3 phenotypically distinct anatomic sites skin, oral mucosa, vermillion form a mucocutaneous junction that can be used for flap prelamination when adequate expansion has occurred, the engineered EVPOME are transferred to the autogenous host for continued differentiation and vascularisation. Immediate viability is maintained by imbibition and inosculation. Kim et al [16] suggested a methodology to functional lip construction. prefabrication and prelamination

01. Two circular punch biopsies: one for skin keratinocytes from posterior auricular area and the other buccal mucosa for oral keratinocytes.

02. Fabrication of mucocutaneous construct $(M / C)$ in vitro and appropriate template for $M / C$ fabrication that would address the size and geometry of the defect.

03. Grafting of ex vivo $M / C$ construct onto the latissimus dorsi muscle (LDM) as a laminate to create an innervated revascularized prefabricated flap. Implantation must be placed parallel to the fibres of LDM as well as parallel to the motor nerve and blood supply based on the thoracodorsal vessels and motor nerve. 
This parallelism will aid in the inset of flap and functional dilation and contraction of the muscle fibres. Location is planned to be just below angle of the scapula for appropriate length of neurovascular bundle.

04. Stoma will be created with in the construct and LDM such that the long axis of the opening is parallel to the muscle fibres to simulate the orbicularis oris muscle.

05. Harvesting of the designer prelaminated flap at 2 to 3 weeks after implantation.

06. Microvascular free transfer to the recipient containing thoracodorsal artery, vein and motor nerve to the External Carotid/Facial artery. External Jugular/Facial vein and a branch of facial nerve. Tark and colleagues showed that it was possible to create a prefabricated skin flap using an acellular dermal matrix in conjunction with cultured keratinocytes. Kim et al in their IPP rat model noticed that underlying muscle integrated completely with the overlying dermal equivalent.

Devices have been developed to assess flap maturation apart from Doppler Examination or surgical exploration. Raman and diffuse reflectant spectroscopy allow for non-invasive detection of graft viability / perfusion through native skin [17]. They use specific wavelength that penetrate overlying skin and maturing prelaminated tissue. The device captures reflectance wavelength which is derived from the concentration of oxy and deoxy $\mathrm{Hb}$ (haemoglobin). The main limitations are delay as it takes about 28 days from the harvest of cell to implantation, cost. There are multiple instances where the duration of fabrication and prelamination would be an issue such as in malignancy.

Cartilage- In craniofacial region, for cartilage engineering, cells with chondrogenic potential like Bone Marrow Derived/periosteum derived mesenchymal cells are used with scaffold such as carbon fibre pads, decalcified bone fibrin glue, collagen gels, alginate hydrogels in presence of FGF-2 (Fibroblast Growth Factor), FGF- $\beta$, bFGF (basic fibroblast growth factor).

Salivary gland- Joraku et al [18] cultivated human salivary gland epithelial cells in polyglutamic acid polymer scaffold. They developed this using an invitro collagen and matrix gel culture system to reconstitute salivary gland tissue and showed functional, differentiated salivary units of acini and
Ducts in 3D construct with production of secretory granules and expression of aquaporin 5 protein.

Nerve tissue engineering- In case of loss of neural function of peripheral nerves, nerve regeneration requires a complex interplay between cells, ECM, growth factors and the guidance of nerve fibres. Neuragen and neurawrapare the approved collagen nerve conduits which serve as guidance channels for treatment of injured nerves [19]. Studies using Schwann cells for tissue engineering nerve constructs were able to obtain improved axonal growth [20].

Tongue regeneration- Cell based reconstruction of the tongue mass was reported in rat model21where myoblast/progenitor cells carried in a collagen gel were implanted into the hemiglossectomized tongue to provide tissue regeneration. The application of cyclic strain to bone marrow-derived stem cells greatly accelerated in vitro skeletal biogenesis to achieve a lined myotube structure.

Condyle- The combination of cartilage tissue progenitor cells carried on a hydrogel scaffold and D.O has been used to reconstruct condylar defect in a goat model. A human shaped condyle was successfully engineered from chondrogenic and osteogenetic rat bone marrow stem cells encapsulated in a biocompatible polymer $[21,22]$.

\section{Molecular Therapy}

The use of molecular therapy in regenerative medicine has led to emergence of research in craniofacial region. Molecular therapy aims at sustained release of growth factors. Dual action of BMP2- gene modified mesenchymal stem cells was introduced by $\mathrm{He} X$ [23].

Molecular therapy also aids in enhancement of endogenous cell mechanism recruitment to regenerate injured bone by local targeting and activation of sphingosine-1 phosphate receptors [24]. The effect of hypoxia induced factor- 1a (HIF 1a) gene on osteogenesis of BMSCs using point mutant technique was explored by Zou et al [25].

Antibody mediated osseous regeneration by Ansari et al [26] assessed the efficacy of newly generated chimeric anti BMP-2 monoclonal antibodies in mediating AMOR (Antibody Mediated Osseous Regeneration) as well as evaluating the sustainability of different biomaterials as scaffolds to participate in AMOR. 


\section{Bio Reactors}

Bioreactors can be defined as devices in which biological or biochemical processes or both are reacted under controlled conditions ( $\mathrm{Ph}$, temperature, pressure, oxygen supply, nutrient supply and waste removal). The ideal bioreactor would need autologous material and scaffold as inputs and after a defined culture periods would output the required amount of the tissue. They are classified as open and closed systems [27]. The types of bioreactors are static culture, steared flask rotating wall, perfusion bioreactors.

\section{Conclusion}

Tissue engineering is definitely the future of reconstructive surgery, the regeneration of simple tissues has been a success, however restoration of complex tissues and its functional restoration are still in the research withstanding challenge.

\section{Reference}

01. Bell E. Tissue engineering- a perspective. J Cell Biochem. 1991 Mar;45(3)239-41.

DOI: $10.1002 / j c b .240450302$ [Crossref]

02. Urist MR, Strates BS. Bone morphogenetic protein. J Dent Res. 1971 Nov-Dec;50(6)1392406.

DOI: $10.1177 / 002$ 20345710500060601 [Crossref]

03. Melek LN. Tissue engineering in oral and maxillofacial reconstruction. Tanta Dental Journal. 2015 Sep 1;12(3)211-23.

[Crossref]

04. Kasper FK, Melville J, Shum J, Wong M, Young S. Tissue engineered prevascularized bone and soft tissue flaps. Oral and Maxillofacial Surgery Clinics. 2017 Feb 1;29(1)63-73.

[Crossref]

05. Schnaper HW, Kleinman HK. Regulation of cell function by extracellular matrix. Pediatr Nephrol. 1993 Feb;7(1)96-104.

[Crossref]

06. Yang S, Leong KF, Du Z, et al. The design of scaffolds for use in tissue engineering- Part I, Traditional factors. Tissue Eng. 2001 Dec;7(6)679-89.

DOI: $10.1089 / 107632701753337645$ [Crossref]
07. Burg KJ, Porter S, Kellam JF. Biomaterial developments for bone tissue engineering. Biomaterials. 2000 Dec;21(23)2347-59.

[Crossref]

08. Costello BJ, Shah G, Kumta $P$, et al. Regenerative medicine for craniomaxillofacial surgery. Oral Maxillofac Surg Clin North Am. 2010 Feb;22(1)33-42.

doi: $10.1016 /$ j.coms.2009.10.09 [Crossref]

09. Fraser JK, Zhu M, Wulur I, et al. Adiposederived stem cells. Methods Mol Biol. 2008;449:59-67.

doi: 10. 1007/978-1-60327-169-1_4 [Crossref]

10. Takahashi K, Tanabe K, Ohnuki M, et al. Induction of pluripotent stem cells from adult human fibroblasts by defined factors. Cell. 2007 Nov 30;131(5):861-72.

DOI: $10.1016 /$ j.cell.2007.11.019 [Crossref]

11. Atala A, Kasper FK, Mikos AG. Engineering complex tissues. Sci Transl Med. 2012 Nov $14 ; 4(160) 160$ rv12.

doi: 10.1126/scitranslmed.3004890 [Crossref]

12. Warnke $\mathrm{PH}$, Springer IN, Wiltfang J, et al. Growth and transplantation of a custom vascularised bone graft in a man. Lancet. 2004 Aug 28-Sep 3;364(9436)766-70.

[Crossref]

13. Heliotis $M$, Lavery KM, Ripamonti $U$, et al. Transformation of a prefabricated hydroxyapati te/osteogenic protein-1 implant into a vascular ised pedicled bone flap in the human chest. Int J Oral Maxillofac Surg. 2006 Mar;35(3)265-9. [Crossref]

14. Lee J, Sung HM, Jang JD, et al. Successful reconstruction of $15-\mathrm{cm}$ segmental defects by bone marrow stem cells and resected autogenous bone graft in central hemangioma. J Oral Maxillofac Surg. 2010 Jan;68(1)188-94. doi: $10.1016 /$ j.joms.2009.08.031 [Crossref]

15. Izumi $\mathrm{K}$, Terashi $\mathrm{H}$, Marcelo $\mathrm{CL}$, et al. Development and characterization of a tissueengineered human oral mucosa equivalent produced in a serum-free culture system. J Dent Res. 2000 Mar;79(3)798-805.

DOI: $10.1177 / 00220345000790030301$ [Crossref] 
16. Kim RY, Bae SS, Feinberg SE. Soft Tissue Engineering. Oral Maxillofac Surg Clin North Am. 2017 Feb;29(1)89-104.

doi: $\quad 10.1016 /$ j.coms.2016.08.007 [Crossref]

17. Khmaladze A, Ganguly A, Kuo S, Raghavan M, Kainkaryam $\mathrm{R}$, Cole $\mathrm{JH}$, et al. Tissue-engineered constructs of human oral mucosa examined by raman spectroscopy. Tissue Eng Part C Methods. 2013;19;299-306.

[Crossref]

18. Joraku A, Sullivan CA, Yoo J, et al. In-vitro recons-titution of three-dimensional human salivary gland tissue structures. Differentiation. 2007Apr;75(4)318-24.

[Crossref]

19. Evans GR, Brandt K, Katz S, et al. Bioactive poly (L-lactic acid) conduits seeded with Schwann cells for peripheral nerve regeneration. Biomaterials. 2002 Feb;23(3)841-8.

[Crossref]

20. Pfister LA1, Papaloïzos $M$, Merkle HP, et al. Nerve conduits and growth factor delivery in peripheral nerve repair. J Peripher Nerv Syst. 2007 Jun; 12(2)65-82.

DOI: $10.1111 /$ j.1529-8027.2007.00125.x [Crossref]

21. Egusa H, Sonoyama W, Nishimura M, et al. Stem cells in dentistry--Part II- Clinical applications. J Prosthodont Res. 2012 Oct;56(4)229-48. doi: 10.1016/j.jpor.2012.10.001 [Crossref]

22. Abou Neel EA, Chrzanowski W, Salih VM, et al. Tissue engineering in dentistry. J Dent. 2014 Aug;42(8)915-28.

doi: $\quad 10.1016 / j . j d e n t .2014 .05 .008 \quad$ [Crossref]
23. He X, Dziak $R$, Mao $K$, et al. Integration of $a$ novel injectable nano calcium sulfate/alginate scaffold and BMP2 gene-modified mesenchymal stem cells for bone regeneration. Tissue Eng Part A. 2013 Feb;19(3-4)508-18.

doi: $10.1089 /$ ten.TEA.2012.0244 [Crossref]

24. Das A, Segar CE, Hughley $B B$, et al. The promotion of mandibular defect healing by the targeting of S1P receptors and the recruitment of alternatively activated macrophages. Biomaterials. 2013Dec;34(38)9853-62.

doi: 10.1016/j.biomaterials.2013.08.015 [Crossref]

25. Zou D, Zhang Z, He J, et al. Repairing criticalsized calvarial defects with BMSCs modified by a constitutively active form of hypoxia-inducible factor-1a and a phosphate cement scaffold. Biomaterials. 2011 Dec;32(36)9707-18.

doi: 10.1016/j.biomaterials.2011.09.005 [Crossref]

26. Ansari S, Moshaverinia A, Pi SH, Han A, Abdelhamid AI, Zadeh $\mathrm{HH}$. Functionalization of scaffolds with chimeric anti-BMP-2 monoclonal antibodies for osseous regeneration. Biomaterials. 2013;34;10191-10198. [Crossref]

27. Depprich R, Handschel J, Wiesmann HP, JäscheMeyer J, Meyer $U$. Use of bioreactors in maxillofacial tissue engineering. British Journal of Oral and Maxillofacial Surgery. 2008 Jul 1; 46(5)349-54.

[Crossref] 